Research Paper

\title{
Effect of Alpha-Ketoglutarate on Growth and Metabo- lism of Cells Cultured on Three-Dimensional Cryogel Matrix
}

\author{
Deepti Singh\#, ${ }^{\#,}$ Tanushree Vishnoi ${ }^{*}$, and Ashok Kumar ${ }^{\bowtie}$ \\ Department of Biological Sciences and Bioengineering, Indian Institute of Technology Kanpur, Kanpur-208016, UP, India. \\ \# Present address: Polymer gel cluster research center, Yeungnam University, 214 Dae-dong, Gyeongbuk 712-749 South Korea. \\ * Authors have equal contribution in this paper.
}

$\triangle$ Corresponding author: Department of Biological Sciences and Bioengineering, Indian Institute of Technology Kanpur, Kanpur-208016, UP, India. Tel.: +91-512-2594051 Fax: +91-512-2594010 Email: ashokkum@iitk.ac.in.

() Ivyspring International Publisher. This is an open-access article distributed under the terms of the Creative Commons License (http://creativecommons.org/ licenses/by-nc-nd/3.0/). Reproduction is permitted for personal, noncommercial use, provided that the article is in whole, unmodified, and properly cited.

Received: 2012.07.31; Accepted: 2012.09.1I; Published: 2013.05.25

\begin{abstract}
Alpha- ketoglutarate $(\alpha-K G)$ is a well-known intermediate of Kreb's cycle and thus one of the important candidates in the role of cellular metabolism. The aim was to investigate the effect of $\alpha-K G$ on the growth and proliferation of different cells (fibroblasts and chondrocytes). Further change in the consumption of glucose and release of ammonia in the media, upon the addition of $\alpha-K G$ was also examined. NIH3T3 and chondrocytes were seeded on two different macroporous cryogel matrices synthesized by poly (hydroxyethylmethaacrylate)-gelatin (HG) and alginate-gelatin (AG). Higher proliferation was observed for both the cell lines in 2-D as well as 3-D where $\alpha-K G$ was added to the media compared to the controls. It was evident that the chondrocytes were metabolically more active in the case of $\alpha-K G$ containing samples as early extracellular matrix (ECM) accumulation was observed. In comparison to the 6-8 weeks duration required by the chondrocytes for ECM accumulation in normal in-vitro culture conditions, $\alpha-K G$ containing samples showed an earlier accumulation within 3 weeks. In order to further validate the results, scanning electron microscopic (SEM) analysis was performed showed high ECM deposition and cells embedded in the matrix. Homogenous distribution of cells on both the synthesized matrix was reported using 4'-6-diamidino-2-phenylindole (DAPI) and propidium iodide (PI) staining. The results clearly showed reduction in ammonia concentration in $\alpha-K G$ containing samples thus leading to decreased ammonia toxicity that builds up during long cell culture conditions thereby enhancing the proliferation and metabolic activity. Thus $\alpha-K G$ can be used potentially for long batch-cultures for the production of vaccines or antibody as well as can play an important role as a bioactive molecule for in vitro neo-cartilage generation.
\end{abstract}

Key words: alpha-ketoglutarate, alginate-gelatin, ammonia toxicity, chondrocytes, cryogel, fibroblasts, poly (hydroxyethyl methacrylate)-gelatin.

\section{Introduction}

One approach in tissue engineering is to use exogenous growth factors and utilize its effect on cell-matrix interactions. There are various growth factors that have been used in enhancing the cell growth and thus decreasing time of neo-tissue formation. For example, bone morphogenetic protein II (BMP II) has shown to enhance the cartilage formation and helps in accelerating cell proliferation. On the 
other hand the advancement in the field of biotechnology has opened up arena for bio-artificial synthesis of therapeutics which includes hormones, vaccines, antibodies and various immuno-adjuvants [1]. One of the most important components for production of these biological products is primary or immortal cells. The cells cultured in laboratory conditions has specific requirements in which the cell media plays an important role [2] but frequent accumulation of toxic catabolic components like ammonia effects the overall cellular growth which directly effects the therapeutic production [3].

There has been constant focus in exploring molecules that show positive effect on overall cellular growth, metabolism and can also help in removing the toxic catabolites. One of such molecules is a-KG, which is a known Kreb's cycle intermediate and plays a critical role in the cellular energy metabolism [4]. MacKenzie and group [5] indicated a-KG as a component for new possible therapy in treating cancers associated with tricarboxylic acid (TCA) cycle dysfunction. Fumarate hydratase (FH) and succinate dehydrogenase $(\mathrm{SDH})$ which are important components of TCA cycle and act as tumor suppressors and loss of either of these components activates a major tumor-supporting event as there is pseudohypoxia condition created in the cells. This event in-turn activates hypoxia-inducible factor (HIF) as HIF-a which is stabilized by $\mathrm{FH}$ and $\mathrm{SDH}$ and excess or defiant TCA cycle components inhibit HIF-a prolyl hydroxylase (PHD). Introduction of cell-permeating a-KG derivatives showed restoration in PHD activity and level of HIF-a to SDH-suppressed cells. a-KG is recognized as a scavenger for ammonia in hepatocyte culture [6] as it serves as a precursor for non-essential amino acid like glutamate and glutamine [7, 8]. Glutamine is found to inhibit degradation of proteins in the muscles and along with transaminated a-KG forms excitatory neurotransmitter glutamate [9]. It is also found to penetrate into the bone tissue and helps in its metabolic regulations [10]. Shuprisha et al. [11] showed real-time assessment of effect of a-KG on organic anion secretion in proximal tubules of perfused rabbit and illustrated that in presence of $a-K G$, the luminal and basolateral sodium ion dicarboxylate $\left(\mathrm{Na}^{+}-\mathrm{DC}\right)$ co-factors could support significantly the net fluorescein (FL) secretion.

It is also reported in few studies that a-KG is involved in collagen metabolism by increasing the activities of prolidase, a known key factor in collagen metabolism [4]. Collagen being important connective tissue in the body which contributes largely towards the structural integrity of the tissues, thereby suggesting that a-KG can be used for controlling the metabolism of the collagen. Effect of a-KG on collagen can make it an important molecule in various therapeutic and cosmetic applications. Other reported beneficial activity of a-KG includes prevention against ischemic injury in heart surgeries [12] and prevention of protein depletion after trauma or surgery [13].

Apart from the media and various growth factors, microenvironment plays an imperative role in cell culture. Major difference in cell behaviour in the culture system and their counter parts in vivo system is the dissociation of cells from three-dimensional (3-D) geometry and their propagation on the two-dimensional (2-D) substrate. The specific cell interaction characteristic of the histology of tissue is lost as cells spread out and starts to proliferate leading to de-differentiation of cells [14]. Culture environment also seems to lack the systemic components that are involved in the homeostatic regulation in the in vivo, as a result cellular metabolism can be more constant in the in-vivo than in-vitro conditions [14]. There are intrinsic factors that need to be added to obtain the cell functions that resemble the in vivo tissue regeneration more closely [15]. Cells have shown to proliferate better in the 3-D environment due to greater available surface area and better transport of nutrients. Progression in the field of tissue engineering has led to development of various scaffolds that are tailor made depending upon their applications.

In our laboratory, scaffolds using natural and synthetic polymers are designed by special approach known as 'cryogelation' $[16,17]$ in which matrices are synthesized at temperature that is normally below the freezing temperature of the solvents. The ice crystals formed during synthesis melt in thawing procedure leaving behind a porous structure known as cryogels. The average pore size of these matrix lies between $50-200 \mu \mathrm{m}$ [18] and the high porosity of the cryogel due to large interconnected pores ensures convective flow of nutrients and mass transport of gases which is unhindered even in long-batch cultures. These cryogels have been successfully used in cartilage tissue engineering [16], cardiac tissue engineering [19], monoclonal antibody productions [20], bioreactors [21] and cell separations [22].

In this study the effect of a-KG on fibroblasts and chondrocytes on two different cryogel matrices such as poly (hydroxyethyl methacrylate)-gelatin (HG) and alginate-gelatin (AG) was studied in order to investigate the effect of a-KG on cellular proliferation in the 3-D scaffold. We have also investigated how the ammonia accumulation and glucose consumption differ in diverse cells in the presence and absence of a-KG. This work was aimed to provide an insight to the role of $\alpha-K G$ as a bioactive molecule in the cell culture and tissue engineering applications. 


\section{Materials and Methods}

Materials: 2-Hydroxyethyl methacrylate (HEMA) (MW: 141.09), sodium salt of alginic acid (from brown algae), gelatin (from cold water fish skin; MW: $\sim 60,000)$, poly(ethylene glycol) diacrylate (PEGDA), Dulbecco's modified Eagle's medium (DMEM), 3-(4,5-dimethylthiazol-2-yl)-2,5-diphenyl tetrazolium bromide (MTT, 98\%) reagent, 4'-6-diamidino-2-phenylindole (DAPI), propidium iodide (PI) and nystatin were all purchased from Sigma Chemical Co (St. Louis, MO, USA). a-KG was supplied by Protista Biotechnology (Lund, Sweden) as a gift. Glutaraldehyde was purchased from s.d. fine-chemicals limited (Mumbai, India). Fetal bovine serum (FBS) and streptomycin-penicillin antibiotic solutions were bought from HyClone (Utah, USA). Ammonium persulphate (APS) was supplied by Merck Chemical Co. (Mumbai, India) and $N, N, N^{\prime}, N^{\prime}$-tetraethylmethylene diamine (TEMED) was purchased from Sisco Research Laboratory (Mumbai, India). All other chemicals used were of analytical grade.

\section{Synthesis of poly (hydroxyethyl methacry- late)-gelatin (HG) cryogel}

Gelatin solution (10 ml; $4 \% \mathrm{w} / \mathrm{v})$ was prepared in degassed water. To this solution hydroxyethylmethacrylate (HEMA) $(0.6 \mathrm{ml})$ with the final concentration of $6 \%(\mathrm{v} / \mathrm{v})$ was added and allowed to cool on ice for a brief period of time. Ammonium persulphate (APS) $(0.1 \mathrm{ml}$ of $10 \% \mathrm{w} / \mathrm{v})$ and TEMED $(0.01 \mathrm{ml})$ were added as initiators along with poly(ethylene glycol) diacrylate (PEGDA) (ratio 1:2) and glutaraldehyde $(25 \% \mathrm{v} / \mathrm{v})$ (ratio 1:20) as cross linkers. The concentration of cross linkers directly depends upon the concentration of polymers. Polymerization reaction was allowed to proceed overnight at $-12{ }^{\circ} \mathrm{C}$ in liquid cryostat (lauda RP1840, Germany). The polymerized gels were washed in de-ionized water at room temperature to remove unpolymerized monomers and were vacuum-dried using a lyophilizer after complete thawing. The dried gels were stored at room temperature until further use.

\section{Synthesis of alginate-gelatin (AG) cryogel}

Alginate $(1 \% \mathrm{w} / \mathrm{v})$ and gelatin $(3 \% \mathrm{w} / \mathrm{v})$ solution was prepared in de-ionized water and were mixed together properly. To this solution $0.2 \%$ glutaraldehyde was added as a crosslinker. The solution was mixed by vortexing and immediately poured in the mould to be kept in the cryostat at $-12 \circ \mathrm{C}$ for $12-16$ $\mathrm{h}$. The samples were then thawed in de-ionized water for 15-20 min and dried using lyophilizer for further use.

\section{Microstructure analysis of cryogels}

Morphology of AG and HG cryogel samples was studied using scanning electron microscopy (SEM, FEI Quanta 200). Scaffolds were dried overnight before gold coating which was performed using a sputter coater. The microscope was operated under high vacuum at $10-20 \mathrm{kV}$ with sample spot size of $4.5 \mathrm{~mm}$ to image the samples. The pore range, pore diameter and interconnectivity of both types of cryogel were determined using scanning electron micrographs associated image analysis software.

\section{Seeding of NIH3T3 on HG and AG cryogels}

Scaffolds were sterilized using ethanol in stepwise gradient concentration $(20 \%-100 \%)$ and equilibrated with PBS $(0.01 \mathrm{M}, \mathrm{pH} 7.0)$ and media. The media was discarded before setting the experiment. To set up the experiment NIH3T3 cells with the cell seeding density of $0.2 \times 10^{5}$ cells/well Dulbecco's modified eagle's medium (DMEM) volume of $1 \mathrm{ml}$ was seeded on AG and HG cryogel scaffolds of $2 \mathrm{~mm}$ thickness. One set of the experiment was performed with $2 \mathrm{mM}$ a-KG containing media (+ve) and another without a-KG in the medium (-ve). Similar protocol was followed for 2-D culture (tissue culture 24 well plate). Experiment was set up for 11 days without changing the medium. For each time point samples in triplicates were set up and the spent medium $(500 \mu \mathrm{l})$ was collected at regular time intervals for glucose and ammonia estimation. MTT assay for cell proliferation was performed on same wells. All the experiments were performed in triplicates.

\section{Isolation of chondrocytes and seeding on cry- ogel matrices}

Articular cartilage from the fresh knee joint of Indian Capra hircus (body weight, 4-6 kg, age, 3-6 months) was used to isolate primary chondrocytes. The fresh knee joint was obtained from the local butcher shop and cartilage was removed from it under aseptic laboratory conditions. Cartilage was minced and subjected to enzymatic digestion with $0.3 \%$ collagenase type II (prepared in serum free DMEM) at $37^{\circ} \mathrm{C}$ for $6 \mathrm{~h}$. The digested cartilage suspension was centrifuged at $150 \mathrm{~g}$ for $5 \mathrm{~min}$. The pellet obtained was then treated with $0.25 \%$ trypsin for 5 min to isolate single cells without clumps or undigested tissue. Trypsin was neutralised by adding $10 \%$ fetal bovine serum (FBS) and the suspension of cells was further centrifuged at $300 \mathrm{~g}$ for $10 \mathrm{~min}$. Cell pellet obtained were first checked for viability by trypan blue vital dye and then cell number was estimated using a haemocytometer. After isolation, cells were cultured to further proliferate and used after first passage. Cell seeding density was same as NIH3T3 
and the experiment was set up for 30 days due to slow proliferation of chondrocytes.

\section{Cellular growth and proliferation on cryogel matrices}

Cryogels and 2-D culture of NIH3T3 and chondrocytes at regular interval were tested for cellular growth and proliferation using 3-(4,5dimethylthiazol-2-yl)-2,5-diphenyl tetrazolium bromide (MTT). MTT assay is spectrophotometeric analysis which quantifies the end product resulting from oxidation of MTT solution by mitochondria of the cells. MTT is regarded as an indirect assay for checking cell growth and proliferation. To describe briefly, the culture medium was collected out from test wells for other biochemical analysis and wells were washed gently with PBS (0.01 M, pH 7.4). MTT solution (0.5\%) containing thiazoyl blue was prepared using serum free DMEM and incubated at $37{ }^{\circ} \mathrm{C}$ for $4 \mathrm{~h}$. The MTT solution was removed carefully and dimethyl sulphoxide (DMSO) was added at a ratio of 3:1 (DMSO to MTT) in each test well to dissolve intracellular formazan crystals and incubated again for $10 \mathrm{~min}$. The blue-violet colour end product was measured by spectrophotometer absorbance at $570 \mathrm{~nm}$.

\section{Estimation of ammonia accumulation}

Ammonia free water was used in the preparation of reagents and washing of glasswares for ammonia estimation. Solution I was prepared by dissolving $1 \mathrm{~g}$ phenol and $5 \mathrm{mg}$ of sodium nitroprusside in $100 \mathrm{ml}$ of degassed water. Solution II was prepared by weighing $250 \mathrm{mg}$ of sodium hydroxide along with $2.158 \mathrm{~g}$ of disodium hydrogen-orthophosphate and $10 \mathrm{ml}$ of sodium hypochlorite and the total volume was made upto $50 \mathrm{ml}$. All the above components were mixed well and tightly capped until further used. The test sample i.e., medium from 3-D and 2-D (+ve* \& -ve $\left.{ }^{\#}\right)$ $(500 \mu \mathrm{l})$ was taken and to this $10 \%$ sodium tungstate and $1 \mathrm{~N}$ sulphuric acid was added to denature protein. The mixture of solution was centrifuged at $1800 \mathrm{~g}$ for $15 \mathrm{~min}$. The supernatant was immediately and carefully collected and mixed with $2.5 \mathrm{ml}$ of solution I and solution II [23]. After thorough mixing, tubes were incubated at $37{ }^{\circ} \mathrm{C}$ for $35 \mathrm{~min}$. The absorbance was recorded at $625 \mathrm{~nm}$ and same protocol was followed for ammonia as standard. All the experiments were done in triplicates.

\section{Estimation of glucose consumption}

Dinitrosalicylic acid (DNS) (10 g) along with 500 $\mathrm{mg}$ of sodium sulphite and $10 \mathrm{~g}$ of sodium hydroxide were mixed in $1000 \mathrm{ml}$ of water. DNS (500 $\mu \mathrm{l})$ and 500 $\mu \mathrm{l}$ of test sample solution i.e., 3-D and 2-D $\left(+\mathrm{ve}^{*} \&\right.$ $\left.-\mathrm{ve}^{\#}\right)$ were added and the tubes were kept at $90^{\circ} \mathrm{C}$ for
15 min to develop red-brown colour complex. The coloured complex developed in the tubes was stabilized using $40 \%$ sodium potassium tartrate (Rochelle salt). The samples were allowed to cool to room temperature and the absorbance was recorded at $575 \mathrm{~nm}$ using a spectrophotometer. All the experiments were done in triplicates.

\section{Scanning electron microscopy (SEM) and staining analysis}

Cryogel scaffolds seeded with chondrocytes were fixed with $2.5 \%$ glutaraldehyde after completely removing the media. Air dried samples were sectioned for SEM analysis and DAPI staining. The SEM of the cryogel scaffolds was performed using same protocol as in case of microstructure analysis of scaffolds. DAPI is known for its affinity to bind with AT rich region of double stranded DNA and is used in regular basis for checking the distribution of cells on scaffolds. The fluorescence emitted upon binding with DNA is observed at excitation wavelength of 350 $\mathrm{nm}$ (Nikon, TE-2000 U). Propidium iodide which is an intercalating dye binds to double stranded nucleic acid by inserting itself between the strands of the DNA or RNA. So any change in the DNA which results in the change in the nucleic acids structure (i.e. increased histone content and nucleosome formation) will reduce the "stainability" of the DNA. The sections of HG and AG scaffolds seeded with NIH3T3 cells were fixed in $2.5 \%$ glutaraldehyde solution for $2 \mathrm{~h}$. After the cell fixation, cells were permeabilized by the buffer $(0.1 \%$ TritonX-100) for $30 \mathrm{~min}$ and then observed under a fluorescence microscope.

\section{Results and Discussion}

\section{Microstructure analysis of cryogels}

The important aspect behind tissue engineering involves culturing of the relevant cells in-vitro into the desired three-dimensional tissue or organ. The 3-D environment provided by the scaffolds to the cells determines the ultimate outcome in tissue engineering applications. The ideal scaffold is expected to be porous matrix with interconnected pores for providing favoured tissue integration and vascularization and should be easily modified or synthesised in different size and shapes. Synthesis of HG and AG scaffolds was carried out under same cryogelation conditions. Both scaffolds retained shapes upon drying at room temperature and showed high water uptake capacity (figure 1 inset digital image). Microstructure analysis by SEM revealed the average pore sizes of HG and AG scaffolds to be $50 \mu \mathrm{m}$ and $66 \mu \mathrm{m}$, respectively (figure 1). Unhindered flow system is again one of the important requirements for tissue engineered 
constructs as it allows convective flow of nutrients and cell waste during the cell culture and facilitates easy exchange of gases. The average flow rate for both cryogels was found to be 4-6 $\mathrm{ml} / \mathrm{min}$ under approximate hydrostatic pressure of $0.01 \mathrm{mPa}$ through $2.5 \mathrm{ml}$ cryogel monoliths column suggesting that the pores of the cryogel matrix were interconnected and did not show any resistance against the solvent. The reason of choosing two different matrix was to determine the effect of a-KG on cells in combination with the 3-D structure or completely independent of the microenvironment provided for cellular growth. Therefore, HG cryogel matrix was chosen due to synthetic polymer component whereas AG was fabricated with natural polymeric materials.

\section{Cellular growth and proliferation on cryogel matrices}

SEM was performed in order to validate the high rate of growth and proliferation observed in MTT assay for the cells seeded on a-KG containing cryogels. Scaffolds allowed uniform and highly dense growth of the cells on a-KG supplied matrices. In ad-

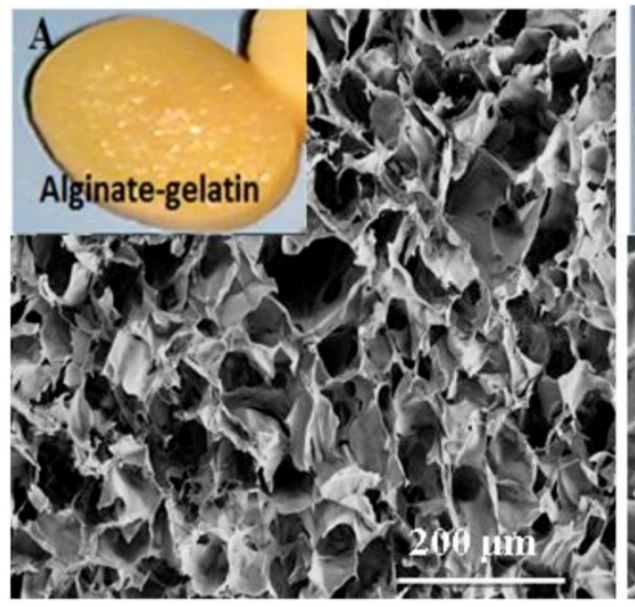

dition ECM was observed on these samples within 3 weeks of culture period (figure 2A inset digital image). These results were further confirmed by SEM analysis (figure 2) indicating that the cells were embedded within the secreted ECM. However, the cells on the matrices without a-KG grew uniformly but ECM secretion was not observed in 3 weeks (figure $2 B$ inset digital image) as it was already reported that in-vitro accumulation of ECM in chondrocytes takes around 6 weeks [24]. The fast ECM secretion is beneficial for cartilage tissue engineering applications. In addition the ECM was observed more in AG scaffold than in HG scaffold which might be because it allowed better cell adhesion, proliferation and growth due to the higher average pore size and different material properties like pore size, structure, surface chemistry of the AG scaffold. Moreover AG scaffolds consisted of natural polymers like alginate compared to HG scaffolds which consists of synthetic polymer, HEMA is already shown to inhibit cell adhesion on its surface because of its hydrophilic nature [16].

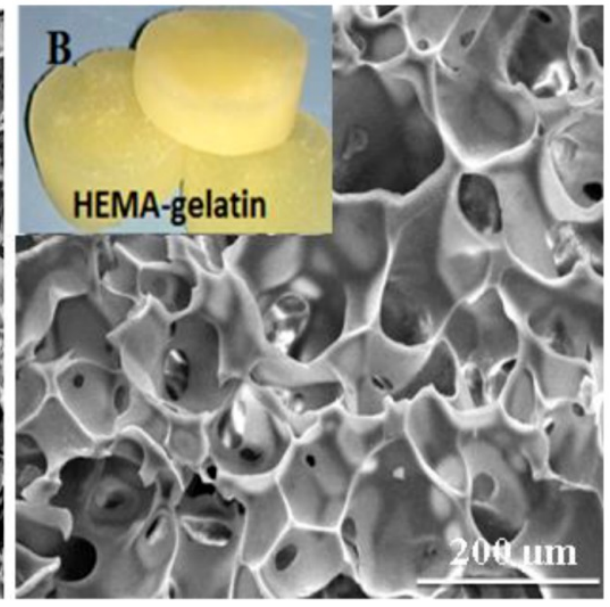

Figure I: Scanning electron microscopic (SEM) images of alginate-gelatin (AG) cryogel (A) and poly (hydroxyethyl methacrylate)-gelatin (HG) (B) showing the pore distribution and interconnected pores (inset digital image of respective water saturated scaffolds).
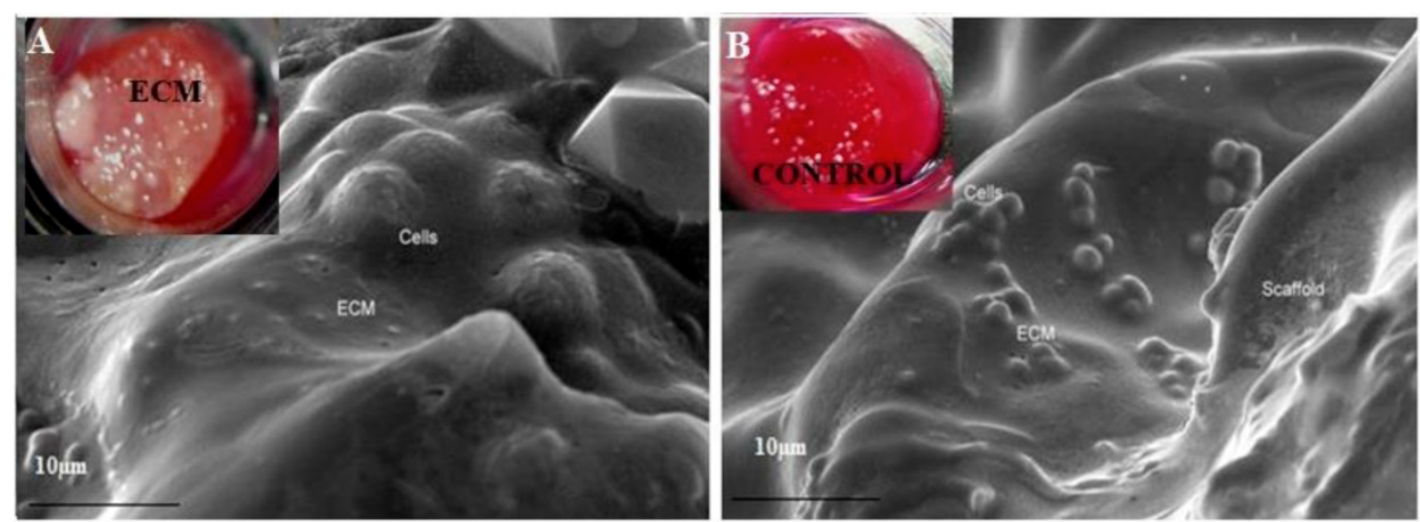

Figure 2: Scanning electron micrograph images of alginate-gelatin (AG) cryogel scaffolds showed visible extra cellular matrix (ECM) deposition during chondrocyte culture. Image A shows cells embedded within the ECM secreted by chondrocytes over the period of 30 days on the AG scaffolds with $\alpha-K G$ (inset digital image of ECM deposition). Image B (control) AG cryogel scaffolds without $\alpha-K G$ (inset digital image of the control). 

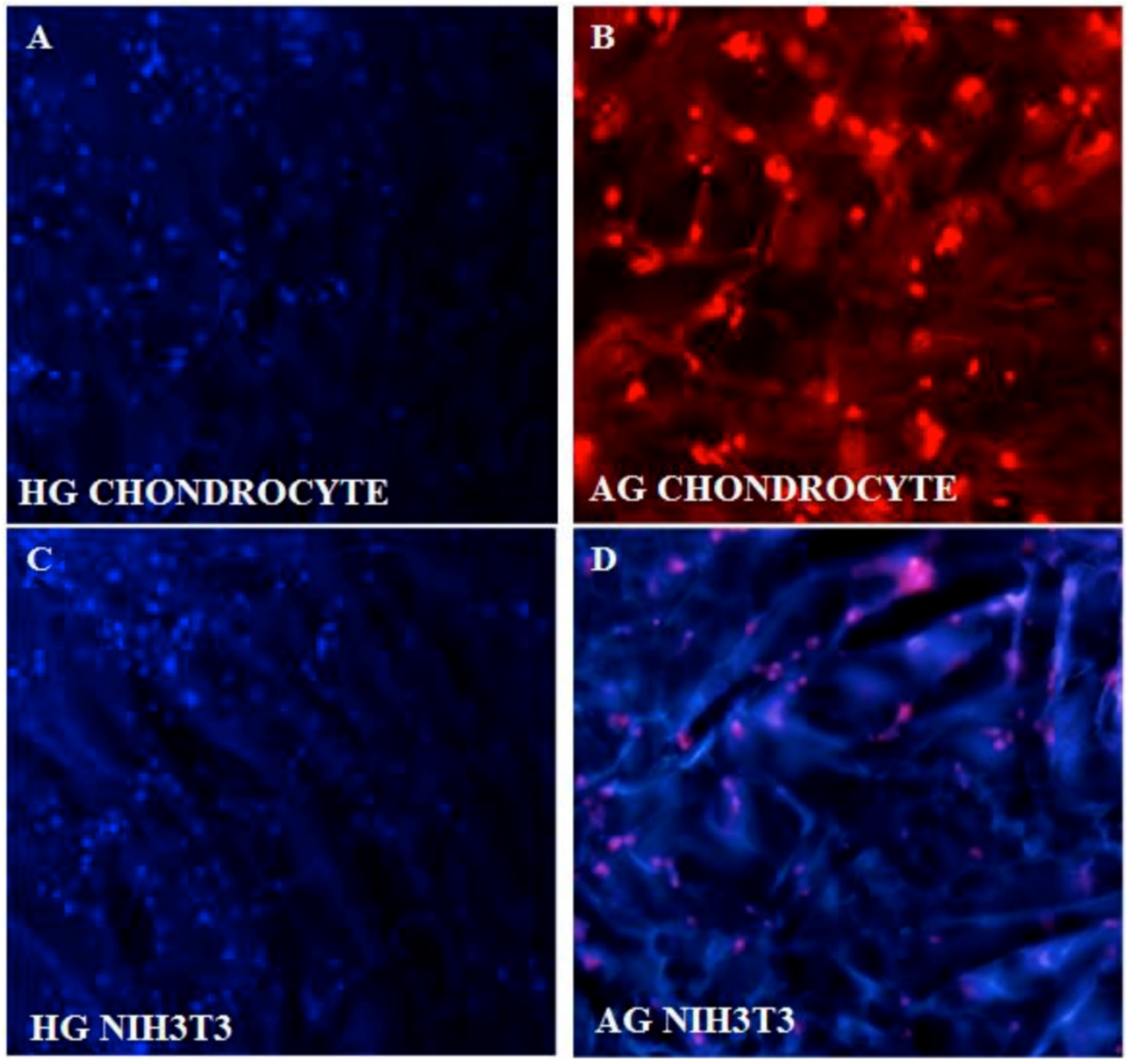

Figure 3: Fluorescent imaging of chondrocytes and NIH3T3 on synthesized cryogel matrices. 4'-6-Diamidino-2-phenylindole (DAPI) image of proliferating chondrocytes on (A) poly(hydroxyethyl methacrylate)-gelatin (HG) and propidium iodide (PI) staining on alginate-gelatin (AG) cryogel (B). Similarly DAPI staining of NIH3T3 fibroblast is shown on both the cryogel matrix of poly(hydroxyethyl methacrylate)-gelatin (HG) (C) and alginate-gelatin (AG) (D).

\section{Matrix influence on cells: cell matrix interac- tion}

DAPI and PI staining were performed as these fluorescent dyes are known for the binding affinity to nucleic acid. DAPI stains nuclei specifically with minimal or no cytoplasm staining and it is known that binding of DAPI with dsDNA produces $\sim 20$ fold fluorescent enhancement and this enhancement results from the water molecules that is being displaced from the DAPI and minor groove of DNA due to the apparent binding effect [25]. PI was performed as the slight difference in $G_{0} / G_{1}$ peak of rapidly dividing cells as opposed by slow dividing cells can be easily distinguished using PI staining. DAPI (figure 3A,B) and PI staining of the cells cultured on a-KG containing samples (figure 3C,D) clearly showed uniform and large number of cells growing throughout the AG and HG scaffolds which is in correlation with the SEM results.

\section{In-vitro cell proliferation assay for NIH3T3 and chondrocytes}

The cell proliferation was studied using the MTT assay for both the cell lines seeded on two different kinds of scaffolds. NIH3T3 cells and chondrocytes were used in our work as previous reports have shown that addition of a-KG inhibit the catabolism of collagen in dermal fibroblasts as well as enhances collagen content via proline synthesis [4]. This could be used as an advantage for culturing chondrocytes as collagen is one of the most important ECM components of cartilage. The samples for MTT assay were collected every alternate day for NIH3T3 cells up to 11 days, whereas on every $5^{\text {th }}$ day up to 30 days for chondrocytes. The optical density of these samples was compared with the control. The cells seeded on a-KG containing media showed a higher rate of proliferation compared to the cells seeded on both in 2-D and 3-D culture in the medium without a-KG. Cells 
seeded on 2-D (tissue culture 24 well plate) reached confluency faster than the 3-D culture due to the availability of lesser surface area (in 2-D) for attachment and proliferation as shown in figure $4(\mathrm{~A}, \mathrm{~B})$. Fibroblasts achieved a higher cell density within $24 \mathrm{~h}$ as compared to chondrocytes because of their faster doubling time and thus attained confluency within 11 days (figure $4 \mathrm{~A}$ ), whereas 21 days were required for chondrocytes to reach confluency (figure 4B) in the 24 well plate. An increment in the cell proliferation was observed in both the cell lines containing a-KG in the medium compared to the control ( $\mathrm{a}-\mathrm{KG}$ negative) as the cell number for both NIH 3T3 and chondrocytes almost doubled in the samples containing a-KG. Comparatively cells seeded on AG scaffold showed slightly higher rate of proliferation compared to HG due to the increased average pore size of AG and also probably because of the favourable material properties which allowed better cell adherence and mass transport of nutrients. So counteracting this slight increase in proliferation on AG scaffold, based on the similar results in 2-D, the increment in cell number can be attributed to the added $\mathrm{a}-\mathrm{KG}$ in the media. $\mathrm{a}-\mathrm{KG}$ is an intermediate in the Kreb's cycle and serves as a source of energy for the cells. Around $50 \%$ of the added $a-K G$ is converted to energy whereas the rest of it is involved in the anabolic effects like synthesis of amino acids [26]. Moreover a-KG was reported to act through $G$ protein-coupled receptors, which are very well known for signalling of the cells [27]. Thus its addition might increase the cell's metabolism and signalling which in turn brings about enhanced cell proliferation. Moreover $\mathrm{a}-\mathrm{KG}$ acts as a scavenger for the ammonia released by the cells in the medium during the culture period. This can minimize the exposure of the cells to the accumulated toxic ammonia and thus allows them to continue to proliferate for a longer period of time [3].

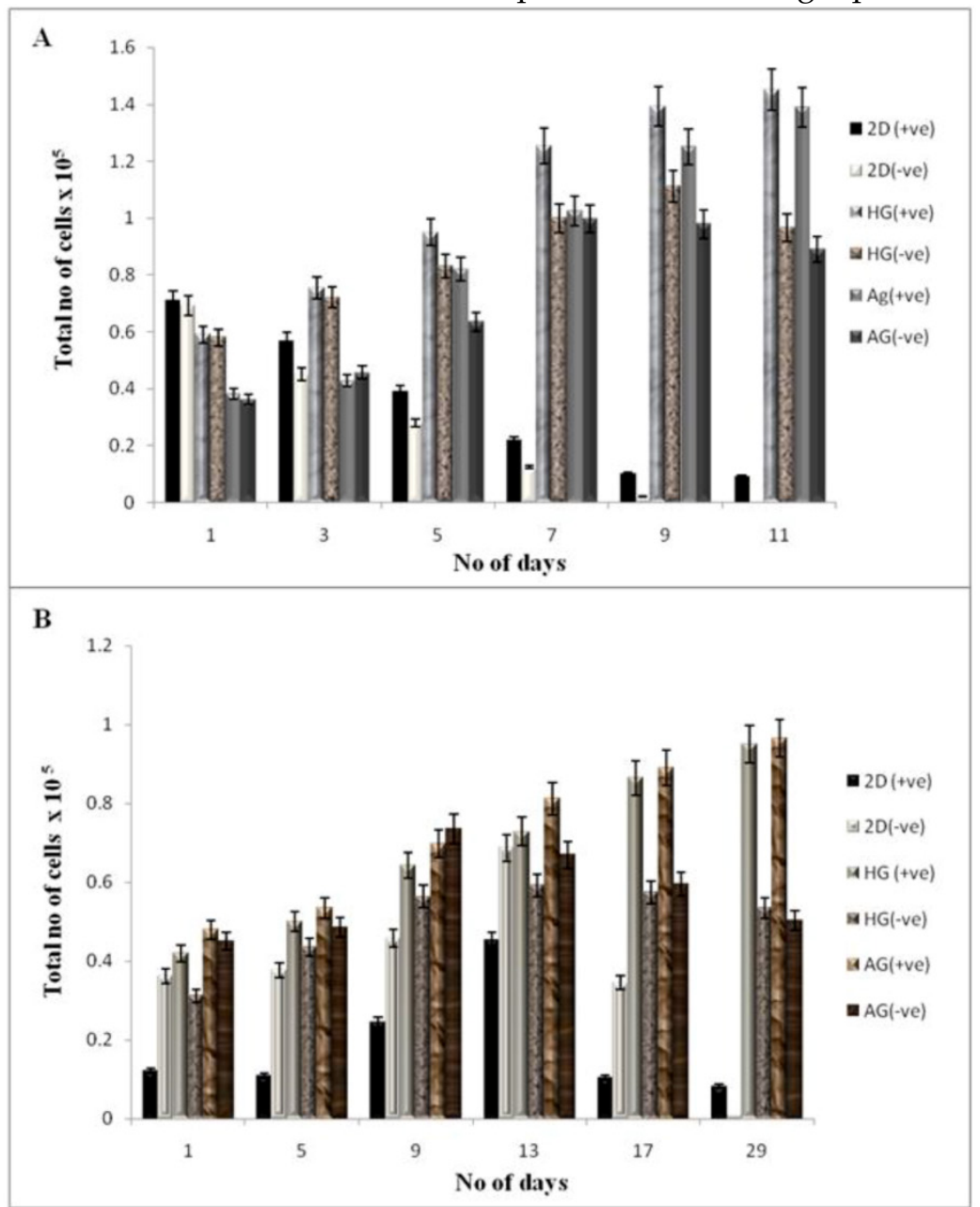

Figure 4: Cell proliferation of NIH3T3 and chondrocytes in 2-D and 3-D culture conditions. HG is poly (hydroxyethyl methacrylate)-gelatin cryogel and AG is alginate-gelatin cryogel. (+ve) indicates experiment with $\alpha-K G$ and (-ve) indicates experiment without $\alpha-K G$. (A) The cellular proliferation of NIH3T3 was measured by (3-(4, 5-Dimethylthiazol-2-yl)-2, 5-diphenyltetrazolium bromide (MTT) assay. The NIH3T3 cells were seeded on two different cryogel scaffolds HG and AG in 3-D along with the 2-D culture. The experiment was divided in two sets; one containing $\alpha-K G$ and the other without $\alpha-K G$ which were analyzed in regular time intervals. The diagram indicates cell proliferation for the samples containing $\alpha-K G$ compared to samples without $\alpha-K G$. (B) Similar experiment was performed for primary chondrocytes. The graph indicates proliferation of chondrocyte in 3-D and 2-D in presence of $\alpha-K G$ as compared to the cells cultured without $\alpha-K G$. All the experiments were performed in triplicates. 


\section{Effect of $\alpha-K G$ on the ammonia accumulation in the media}

Extended hyperammonaemia condition has been reported to result in coma and convulsions [28]. The results clearly show here the reduction in the concentration of ammonia released in the media in a-KG containing cultures. Similar results were observed in our previous study in which a-KG showed to enhance cell proliferation by acting as scavenger for toxic ammonia, thereby increasing the monoclonal antibody production in the in-vitro system [29]. The supernatant from both the 2-D and the 3-D samples were collected for ammonia analysis before performing MTT assay. The samples were then analysed for presence of ammonia by the indo-phenol reaction. The absorbance of the coloured product was read at $490 \mathrm{~nm}$. Ammonia standard was taken in 0, 0.5, 1.0 $\mathrm{mM}$ concentration to obtain standard curve, which was further used in determining the ammonia concentration in test sample. The toxic concentration of ammonia for the cells has been reported to be 1-10 $\mathrm{mg} / \mathrm{dl}$ at which cells enter the stationary phase and leading to apoptosis [30]. In both 2-D and 3-D (fibroblast and chondrocyte) culture, the a-KG containing samples showed decreased amount of ammonia in the medium compared to the samples without a-KG during the culture period (figure 5A, B). Moreover chondrocytes are slow growing cells compared to fibroblasts as a result of which the ammonia accumulation is slow in case of chondrocytes over the period of time. This might be because a-KG is known to maintain the $\mathrm{N}_{2}$ balance. It does so by using the released ammonia from the medium to recycle amino acids like glutamine and glutamate. It serves as a precursor for glutamine, a non-essential amino acid. This conversion is well established and is as follows [31]:

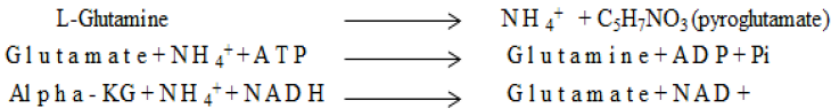

The synthesized glutamine is a known metabolic fuel for the cells and also a precursor for the synthesis of glutamate, a known neurotransmitter. We observed here that $\mathrm{a}-\mathrm{KG}$ helps in the enrichment of the medium and at the same time causes reduction of the build-up toxicity leading to enhanced proliferation and survival of the cells over the period of time.

\section{Glucose consumption in the presence of $\alpha-K G$}

Glucose is one of the main sources of energy for the cells to carry out various metabolic activities. So in order to check the effect of a-KG on cells and their proliferation, glucose measurement using DNS method was performed. The standard graph was used to determine the concentration of the glucose in the test samples as well as the control. Compared to the control as shown in figure 6 the glucose consumption decreased for the a-KG containing samples. It is known that $\mathrm{a}-\mathrm{KG}$ undergoes transamination to amino acids like glutamate and glutamine wherein glutamate is a known neurotransmitter and glutamine, a precursor for glutamate, can also serve as an alternate source of energy for the cells when present with sugars like glucose. Glutamine undergoes aerobic oxidation from TCA cycle resulting in cell energy. Thus the samples containing a-KG resulted in less glucose consumption compared to the control [32]. Similar results were observed for glucose consumption in a-KG containing 2-D cultures.

In conclusion our finding observes the positive effect of a-KG on different cells in 3-D culture. In this study it was clear that enhanced cell proliferation could be achieved by adding a-KG in the culture medium. In our previous study improved production of monoclonal antibody was achieved by the addition of a-KG in medium [2]. Macroporous cryogel matrix helped in the better nutrient transport and cell growth and addition of a-KG prevented the accumulation of the toxic catabolites resulting in improved culture stability. In chondrocyte culture in 3-D environment, the ECM secretion was fast and started accumulation of the matrix within three weeks of the culture time which was well ahead than normal in vitro ECM production. Thus this provides the understanding that a-KG can be used as a key ingredient in enhancing the cell growth and proliferation in general and for cartilage tissue engineering in particular. The effect of a-KG on both types of cells was positive however was not independent of the matrix and microenvironment. Cells cultured on AG showed better proliferation in comparison to HG and similar trend was noted even with ammonia and glucose. There is inter-dependency between the factors and scaffold and interplay between these moieties results in enhanced cell proliferation and increased ECM production. The a-KG can be used potentially as growth factor especially in the field of cartilage tissue engineering. Chondrocytes are slow growing cells as a result of which ammonia toxicity builds up more often leading to slower formation of neo-cartilage hence various approaches are needed to overcome this limitation. We have shown the use of a-KG at certain concentration could overcome this bottleneck in cartilage tissue engineering for enhanced cell growth and proliferation and for negating the toxic effect of ammonia to obtain faster secretion of matrix component leading to the early formation of neo-cartilage can be a cost-effective substitute as a bioactive molecule. 




Figure 5: Ammonia measurements in NIH3T3 and chondrocyte cultures in 2-D and 3-D conditions. HG is poly (hydroxyethyl methacrylate)-gelatin cryogel and $A G$ is alginate-gelatin cryogel. (+ve) indicates experiment with $\alpha-K G$ and (-ve) indicates experiment without $\alpha-K G$. Effect of $\alpha-K G$ on ammonia accumulation in NIH3T3 cells and chondrocytes derived by standard graph $\left(0,0.5,1.0 \mathrm{mM}\right.$ concentration) was plotted to derive the $\mathrm{R}^{2}$ value, which was further used in determining the ammonia concentration in test samples. (A) The ammonia accumulation in NIH3T3 cultured in presence of $\alpha-K G$ in 3-D ( $H G, A G)$ and 2-D conditions in comparsion to expereiment set-up without $\alpha-K G(-v e)$. (B) Figure shows the concentration of the ammonia accumulated in the media when chondrocytes were cultured in the presence and absence of $\alpha-K G$ for a period of 29 days. All the experiments were performed in triplicates.

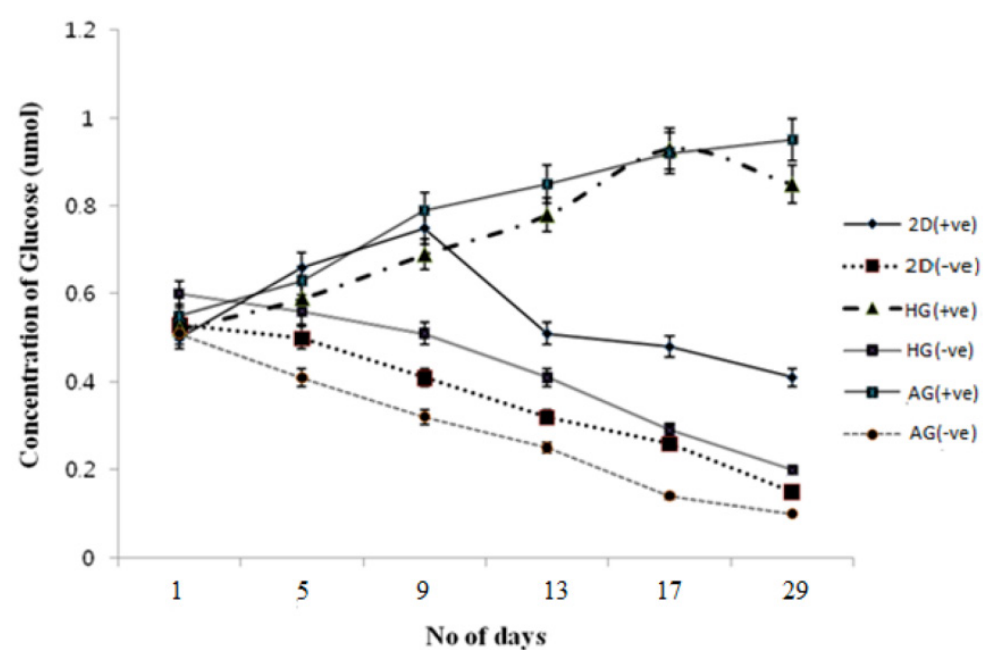

Figure 6: Glucose metabolism during chondrocyte culture in 2-D and 3-D culture conditions in the presence and absence of $\alpha-K G$. HG is poly (hydroxyethyl methacrylate)-gelatin cryogel and AG is alginate-gelatin cryogel. (+ve) indicates experiment with $\alpha-K G$ and (-ve) indicates experiment without $\alpha-K G$. Effect of $\alpha-K G$ on the chondrocyte was checked over a period of 3 weeks in two different cryogel scaffolds. Standard graph was obtained by plotting the known concentration of glucose against the absorbance which was further used to determine the glucose concentration in the test samples. All the experiments were performed in triplicates. 


\section{Acknowledgement}

Authors would like to acknowledge the financial support by Department of Biotechnology (DBT), Govt of India and Department of Science and Technology (DST), New Delhi- through its Unit of Excellence on Soft Nanofabrication at IIT Kanpur. TV would like to acknowledge Council of Scientific and Industrial Research for Sr. Research Fellowship.

\section{Abbreviation}

Alpha- ketoglutarate: a-KG; poly (hydroxyethylmethaacrylate)-gelatin: HG; Alginate-gelatin: AG; Extracellular matrix: ECM; Scanning electron microscopic: SEM; (3-(4,5-Dimethylthiazol-2-yl)-2,5diphenyltetrazolium bromide: MTT; 4'-6-diamidino2-phenylindo le: DAPI; Propidium iodide: PI.

\section{Competing Interests}

The authors have declared that no competing interest exists.

\section{References}

1. Rudra JS, Tian YF, Jung JP, and Collier JH. A self-assembling peptide acting as an immune adjuvant. Proc Natl Acad Sci USA. 2010; 107: 622-627.

2. Nilsang S, Kumar A, and Rakshit SK. Effect of a-Ketoglutarate on monoclonal antibody production of hybridoma cell lines in serum-free and serum-containing medium. Appl Biochem Biotechnol. 2006; 151: 489-501.

3. Nilsang S, Nehru V, Plieva FM, Selva NK etal. Three-dimensional culture for monoclonal antibody production by hybridoma cells immobilized in macroporous gel particles. Biotechnol Prog. 2008; 24: 1122-1131.

4. Son ED, Cho GH, Kim H, Lee B, et al. Alpha-ketoglutarate stimulates procollagen production in cultured human dermal fibroblasts and decreases UVB induced wrinkle formation following topical application on the dorsal skin of hairless mice. Biol Pharm Bull. 2007; 30: 1395-1399.

5. MacKenzie ED, Selak MA, Tennant DA, Payne L, et al. Cell-Permeating a-Ketoglutarate Derivatives Alleviate Pseudohypoxia in Succinate Dehydrogenase-Deficient Cells. Mol Cell Biol. 2007; 27: 3282-3289.

6. Rutten E P A. Skeletal muscle glutamate metabolism in health and disease: state of the art. Curr Opin Clin Nutr Metab Care. 2005; 8: 41-51.

7. Sheu KF, and Blass JP. The alpha-ketoglutarate dehydrogenase complex. Ann NY Acad Sci. 1999;893: 61-78.

8. Nissim I. Newer aspects of glutamine/glutamate metabolism: the role of acute $\mathrm{pH}$ changes. Am J Physiol. 1999; 277: 493-497.

9. Gibson GE, Park LC, and Sheu KF. The alpha-ketoglutarate dehydrogenase complex in neurodegeneration. Int J Neurochem. 2000; 37: 97-112.

10. Berkich A D, Xu Y, LaNoue FK, Gruetter R, and Hutson SM. Evaluation of brain mitochondrial glutamate and alpha-ketoglutarate transport under physiologic conditions. J Neurosci Res. 2005; 79: 106-113.

11. Shuprisha A, Lynch RM, Wright SH, and Dantzler WH. Real-time assessment of a-ketoglutarate effect on organic anion secretion in perfused rabbit proximal tubules. Am J Physiol Ren Physiol. 1999; 277: 513-523.

12. Peuhkurinen KJ, Takala TE, Nuutinen EM, and Hassinen IE. Tricarboxylic acid cycle metabolites during ischemia in isolated perfused rat heart. Am J Physiol. 1983; 244: 281-288.

13. Wernerman J, Hammarqvist F, and Vinnars E. Alpha-ketoglutarate and post-operative muscle catabolism. Lancet. 1990; 335: 701-703.

14. Vunjak-Novakovic G, Freshney RI. Culture of Animal Cells: A Manual of Basic Technique. Germany: Wiley-VCH. 2005.

15. Albert B, Bray D, Lewis J, Raff M, et al. Molecular Biology of the Cell, $3^{\text {rd }}$ edition. New York: Garland Publishing. 1994.

16. Singh D, Tripathi A, Nayak V, and Kumar A. Proliferation of chondrocytes on a 3-D modelled macroporous poly(hydroxyethylmethacrylate)gelatin cryogel. J Biomater Sci Polymer. 2011; 22: 1733-1751.

17. Kumar A, Mishra R, Reinwald Y, and Bhat S. Cryogels: Freezing unveiled by thawing. Materials Today. 2010; 13: 42-44.
18. Jain E, Srivastava A, and Kumar A. Macroporous interpenetrating cryogel network of poly(acrylonitrile) and gelatin for biomedical applications. J Mater Sci Mater Med. 2009; 20: S173-179.

19. Singh D, Nayak V, and Kumar A. Proliferation of myoblast skeletal cells on three dimensional supermacropous cryogel. Int J Biol Sci. 2010; 6: 371-381.

20. Jain E, Karande AA, and Kumar A. Supermacroporous polymer-based cryogel bioreactor for monoclonal antibody production in continuous culture using hybridoma cells. Biotechnol Prog. 2010; 27:170-180.

21. Bansal V, Roychoudhury PK, Mattiasson B, and Kumar A. Recovery of urokinase from integrated mammalian cell culture cryogel bioreactor and purification of the enzyme using p-aminobenzamidine affinity chromatography. J Mol Recogn. 2006; 19:332-339.

22. Kumar A, and Srivastava A. Cell separation using cryogel based affinity chromatography. Proc Natl Acad Sci USA. 2010; 5: 1737-47.

23. Murry MM. The Estimation of Ammonia and Urea in Blood and Urine. J Biochem. 1925; 19: 294-299.

24. Freed LE. Neocartilage formation in-vitro and in-vivo using cells cultured on synthetic biodegradable polymers. J Biomed Mater Res. 1993; 27: 11-23.

25. Barcellona ML, Cardiel G, and Gratton E. Time-resolved fluorescence of DAPI in solution and bound to polydeoxynucleotide. Biochem Biophys Res Commun. 1990; 170: 270-280.

26. Harrison AP, and Pierzynowsk f. Biological effects of 2-oxoglutarate with particular emphasis on the regulation of protein, mineral and lipid absorption/metabolism, muscle performance, kidney function, bone formation and cancerogenesis, all viewed from a healthy ageing perspective state of the art- review article. J Physiol Pharmac. 2008; 58: 91-106.

27. Miao FJ, Lin DC, Schwandner RT, Wang Z, et al. Citric acid cycle intermediates as ligands for orphan G-protein-coupled receptors. Nature. 2004; 429 :188-193.

28. Takanashi J, Barkovich J, Cheng SF, Kostiner D, et al. Brain MR Imaging in Acute Hyperammonemic Encephalopathy Arising from Late-Onset Ornithine Transcarbamylase Deficiency. Am. J. Neuroradiol. 2003; 24: 390-393.

29. Nilsang S, Selva NK, Galaev I, Rakshit SK, et al. Monoclonal antibody production using a new supermacroporous cryogel bioreactor. Biotechnol Prog. 2008; 23: 932-939.

30. Klucinski W, and Targowski SP. Ammonia toxicity for mammalian and avian lymphocytes from blood. Immunopharmacol. 1984; 8: 47-52.

31. Haghighat $\mathrm{N}$, and McCandless DW. Effect of ammonium chloride on energy metabolism of astrocytes and C6-glioma cells. In-Vitro Metab Brain Dis. 1997; 12:287-298.

32. Rajendra Y, Kiseljak D, Baldi L, Hacker DL, et al. Influence of glutamine on transient and stable recombinant protein production in $\mathrm{CHO}$ and HEK-293 cells. BMC Proceedings. 2011; 5: 35-37. 